\title{
Co-rotating Photospheric Clouds in $\mu$ Centauri
}

\author{
L.A. Balona
}

South African Astronomical Observatory, PO Box 9, Observatory 7935, Cape Town, South Africa

D. James

School of Physics and Astronomy, University of St. Andrews, Scotland

W.A. Lawson

School of Physics, University College UNSW, Australian Defence Force Academy, Canberra ACT 2600, Australia

R.R. Shobbrook

Chatterton Astronomy Dept, School of Physics, University of Sydney, Australia

\begin{abstract}
Several periods close to $0.5 \mathrm{~d}$ have been reported for $\mu$ Cen. We obtained over 300 echelle spectra and simultaneous multicolour photometry over a two-week period. We find that the period is $0.66 \mathrm{~d}$ and is, in fact, singly periodic. Greyscale images of many consecutive nights show moving features which do not repeat nightly, as may be expected for $\mathrm{P}=0.5 \mathrm{~d}$, but repeat approximately every alternate night. In spite of this, periodogram analysis of the radial velocity, as measured at the point of minimum intensity in the spectral line, does indeed show a 0.5 -d period. The amplitude of this periodicity decreases during the course of the run. This seeming contradiction, as well as the apparent multiperiodicity, is due to the use of period finding techniques which exclude singly-periodic solutions of a complex nature. We interpret the variations as due to two, co-rotating, circumstellar clouds distributed around the star, such that they are separated by a time interval of $0.5 \mathrm{~d}$. It appears that $\mu$ Cen is another example of the growing list of Be stars in which the periodicity is due to co-rotating photospheric clouds.
\end{abstract}

\section{Introduction}

Rivinius et al. (1998) find that the radial velocity of $\mu$ Cen (B2IV-Ve, $v \sin i=$ $175 \mathrm{~km} \mathrm{~s}^{-1}$ ) is multiperiodic. There are four periods, all within $2 \%$ of $P=$ $0.505 \mathrm{~d}$ and another two periods near $0.28 \mathrm{~d}$. If these periodicities can be confirmed, it proves beyond doubt that nonradial pulsations (NRP) are present in this star. 


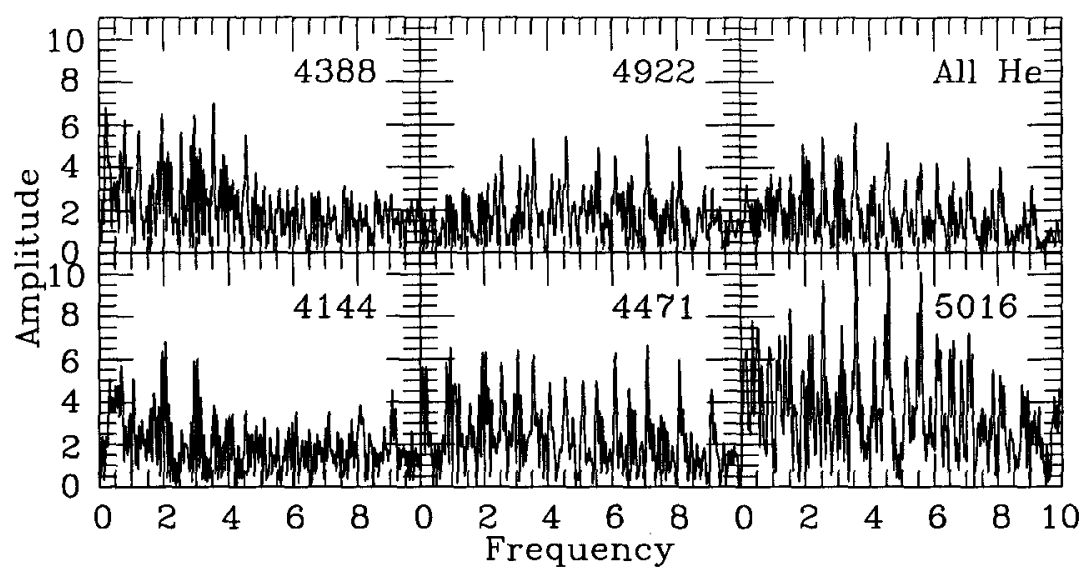

Figure 1. Periodograms of the position of minimum intensity (the "radial velocity") of individual $\mathrm{He}$ I lines and of the mean of all helium lines. The frequency is in cycles/day and the amplitude in $\mathrm{km} \mathrm{s}^{-1}$.

We obtained over 300 echelle spectra of $\mu$ Cen during a continuous twoweek observing run on the SAAO $1.9-\mathrm{m}$ reflector. This was supplemented by echelle spectra obtained at Mt Stromlo. In addition, Strömgren photometry was obtained over the same period using the $0.5-\mathrm{m}$ reflector at SAAO and the 24 -in reflector at Siding Springs. Most of the nights at SAAO were usable, but bad weather in Australia prevented the full coverage that we had hoped to obtain. In this preliminary note, only the SAAO data are used.

The following lines were analyzed: He I lines at 4144, 4388,4471, 4922, $5016 \AA$; Si III 4553 line; $\mathrm{C}$ II 4267 and the $\mathrm{H} \beta$ and $\mathrm{H} \gamma$ lines. Radial velocities, obtained by fitting the core of the line around minimum intensity and also from the centroid of the line were calculated. A standard periodogram analyses of these radial velocities did not show any strong periodicities. Some examples are shown in Fig. 1. It is clear from the figure that there is no obvious dominant periodicity, as measured by this standard technique. The $2.0 \mathrm{~d}^{-1}$ frequency obtained by Rivinius et al. (1998) is present in many of the periodograms, but is usually not the strongest peak except in He 4144 and Si III 4553.

The periodograms of Fig. 1 can be understood when it is realized that at the time of our observations the amplitude of the $0.5-\mathrm{d}$ periodicity was below average (Rivinius; private communication). Indeed, it is at a minimum on the last few nights of our run. If we use only the first few nights of our data, the 0.5-d periodicity appears quite clearly, confirming that the amplitude is high at the beginning of the run. We feel, however, that another interpretation is possible.

\section{The period}

Notice that there are implicit assumptions in using standard Fourier techniques: (i) the radial velocity variations are approximately sinusoidal and (ii) the posi- 

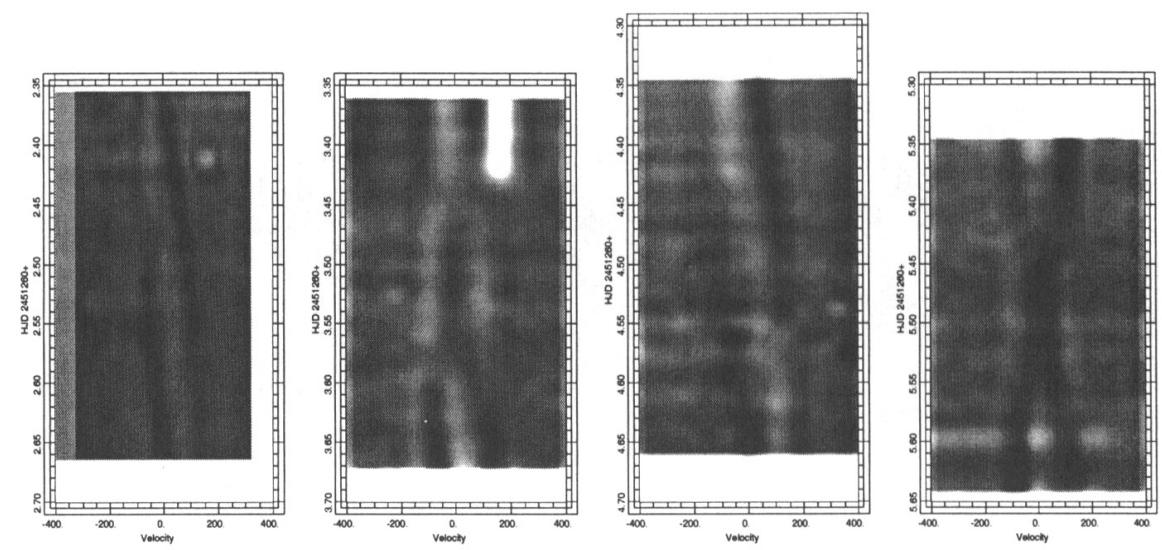

Figure 2. Greyscale images of stacked difference spectra of the mean of five helium lines on four consecutive nights. Dark means absorption; the total range is about 5 percent. The bright red wing emission at the start of the second night occurs only in He I 4144

tion of line minimum is a measure of a physical quantity. In the pulsation model, both these assumptions are justified, but if they are not correct we would be restricting our solutions and possibly missing the correct solution. One needs to consider the possibility that the variations in $\mu$ Cen may be due to a complex rotating surface pattern as found in $\eta$ Cen (Balona 1999), for example, and for which the same difficulties occur in the periodograms.

We calculated "difference" spectra for all the lines by dividing each spectrum by the mean line profile. In this way, small variations are more easily seen. We then stacked these difference spectra as a function of time and created greyscale images for each night. In Fig. 2 we show stacked difference spectra for four consecutive nights at the beginning of our run. During these nights the amplitude of the 0.5-d radial velocity periodicity is predicted to be about 6 $8 \mathrm{~km} \mathrm{~s}^{-1}$, somewhat below the average value of $12 \mathrm{~km} \mathrm{~s}^{-1}$ (Rivinius, private communication).

We notice that the images are quite different on consecutive nights. If the period is close to $1.0 \mathrm{~d}$ or $0.5 \mathrm{~d}$, we would expect to see the same pattern repeated at almost the same time every night; instead the pattern seems to repeat every two nights. Notice, also, the very clear red to blue moving feature on alternate nights, not reported in Rivinius et al. (1998). Inspection of all 13 greyscale images, most of them covering 8 hours of continuous observations, confirms the pattern shown in Fig. 2. Therefore, the star must be singly periodic with a period of about 2 days or a submultiple thereof $(P \approx 2,1,2 / 3,1 / 2, \ldots$ days $)$. We can rule out periods shorter than $2 / 3 \mathrm{~d}$ because this would lead to repeatability on consecutive nights, which we do not see. Therefore we are left with two possible periods, $P \approx 2 \mathrm{~d}$ or $P \approx 2 / 3 \mathrm{~d}$. We examined greyscale difference spectra for several values close to these two periods. We found the most likely period to be $P=0.66 \mathrm{~d}$. 
Cuypers, Balona \& Marang (1989) obtained photometry from South Africa and Chile. No convincing period was found, but a frequency of either $0.48 \mathrm{~d}^{-1}$, or $1.48 \mathrm{~d}^{-1}$, was present during the 1987 season at SAAO. The latter is the same as the spectroscopic period found above. We suspect the true period to be $P=0.67 \mathrm{~d}$. There is no sign of the $0.5-\mathrm{d}$ period in these data. We obtained simultaneous uvby photometry of $\mu$ Cen using HR 5063 and HR 5439 as local comparisons. No obvious periodicity can be obtained from these data. There is no power at $P=0.50 \mathrm{~d}$, though there is some evidence of the $0.66-\mathrm{d}$ period after removing the trend.

\section{Discussion}

Intensive high dispersion observations of $\mu$ Cen show that the line profile variations at this epoch can be described by a single period, most probably $P=$ $0.66 \mathrm{~d}$. It is clear that a single 0.5 -d period will not reproduce the variations seen in Fig. 2. Nor can a single 0.28-d period (the other group of multiperiodicities reported by Rivinius et al., 1998), as this will lead to more than a complete cycle in the 8-hour runs shown in Fig. 2. A combination of a 0.5 -d and 0.28-d periods should show two strong peaks in the periodogram, which is not observed. Note, also, that one cannot recognize the red-to-blue moving feature in the radial velocities. Red-to-blue moving subfeatures are not mentioned by Rivinius et al. (1998).

Inspection of greyscale images of difference spectra phased with the 0.66 -d period shows that there are two distinct sinusoidal variations of excess emission flanked by absorption wings. Our preliminary interpretation is that these are two gas clouds seen close to the photosphere. The angle of inclination of the star is intermediate and we see the beyond-the-pole motion of the two clouds, giving rise to red-to-blue movement of the subfeatures during some phase intervals. The two clouds are not spaced diametrically opposite each other, but such that the time interval between meridian crossings is $0.50 \mathrm{~d}$. The unequal spacing of the clouds produces a strongly non-sinusoidal variation over the period of rotation. We believe this to be responsible for the apparent multiperiodicity. Note, also, that most of the HEROS data on which the analysis of Rivinius et al. (1998) is largely based, consists of one observation per night.

There is a steady reduction in the emission intensity of one of the clouds during the course of the run. The cause of this long-period intensity variation, which is somehow connected with emission-line outbursts, needs to be investigated. The star may be the most important key to our understanding of outbursts in Be stars.

\section{References}

Balona, L.A., 1999, MNRAS 306, 407

Cuypers, J., Balona, L.A., Marang, F., 1989, A\&AS 81, 151

Rivinius, Th., Baade, D., Štefl, S., Stahl, O., Wolf, B., Kaufer, A., 1998, A\&A 336,177 